\title{
A Extensão Universitária e a importância de processos participativos em saúde mental
}

The university extension and the importance of participatory processes in mental health

\section{RESUMO}

Este estudo de abordagem qualitativa, elaborado com recursos metodológicos das pesquisas bibliográfica e documental, contempla os resultados de um projeto de extensão universitária empreendido no âmbito da Rede de Atenção Psicossocial (RAPS) no Distrito Federal, Brasil, no período de agosto de 2018 a dezembro de 2019. Nesse projeto, adotou-se o suporte conceitual da

\footnotetext{
${ }^{1}$ Assistente Social, mestre em Saúde Púbica(UFSC), doutora em Serviço Social (PUC/SP). Docente do Departamento de Serviço Social e do Programa de Pós-Graduação em Política Social da Universidade de Brasília(UnB), DF, Brasil. Telefone: 5561981080161. E-mail: andreiaoliveirasus@gmail.com.

${ }^{2}$ Enfermeira, Mestre em Enfermagem Psiquiátrica(USP) e Doutora em Ciências da Saúde (UnB). Docente do Departamento de Enfermagem da Universidade de Brasília(UnB) e membro da equipe do Programa de Atendimento ao Alcoolismo do Hospital Universitário de Brasília/DF. E-mail: mariagussi@gmail.com ${ }^{3}$ Bióloga, mestre em Ciências Sociais em Saúde (University of Manchester, Inglaterra) doutora em Educação (UFSCar/SP). Docente do Departamento de Saúde Coletiva da Universidade de Brasília(UnB), DF, Brasil.E-mail: modeh@unb.br

${ }^{4}$ Assistente Social e Mestre em Serviço Social (UFSC), doutoranda em Política Social pelo Programa de Pós-Graduação em Política Social da Universidade de Brasília(UnB)/DF. E-mail:

fernanda.scalzavara@gmail.com

${ }^{5}$ Terapeuta ocupacional, mestre em Enfermagem Psiquiátrica (USP/EERP) doutora em Psicologia Clínica e Cultura (UNB/DF). Docente do curso de Terapia Ocupacional da Faculdade de Ceilândia da Universidade de Brasília(UnB)/DF, Brasil. E-mail: ioncampos2016@gmail.com

${ }^{6}$ Enfermeira, Mestre em Enfermagem Psiquiátrica e Doutora em Enfermagem (EERP/USP). Docente do Departamento de Enfermagem e do Programa de Pós-Graduação em Bioética da Universidade de Brasília (UnB)/DF. E-mail: limamgbs@gmail.com
} 
pesquisa-ação, a perspectiva metodológica compartilhada de atuação, composta por interação social e colaboração entre os distintos sujeitos envolvidos na ação extensionista, com ênfase no protagonismo e participação social de usuários da RAPS, seus familiares e profissionais de saúde. $\mathrm{Na}$ interpretação dos resultados da ação extensionista, foram realçados os significados políticos identificados na relação entre a atuação dos sujeitos envolvidos e suas vivências da vida cotidiana. Dessa maneira, a prática da extensão universitária pôde propiciar aos acadêmicos e profissionais de saúde, mobilizados pelo contato real com expressões da questão social e pela reflexão teórica, a democratização do saber e a formação com senso crítico e ético, com o objetivo de contribuir de modo mais qualificado para o fazer dos serviços de atenção em saúde mental. Aos usuários e familiares, a aproximação da prática da extensão se mostrou um importante incentivo para fortalecer o protagonismo nos espaços de atenção, cuidado e participação social na política pública de saúde mental.

PALAVRAS-CHAVE: Extensão comunitária, Relações comunidade-instituição, Serviços de saúde mental, Participação social.

\section{ABSTRACT}

This qualitative approach study, drawn up with methodological resources from bibliographic and documentary researches, contemplates the results of a university extension project carried out within the scope of the Psychosocial Care Network (RAPS) in the Federal District, Brazil, from August 2018 to December 2019. This project chose the conceptual support of research-action, the methodological perspective shared of operation, formed by social interaction and collaboration between the different subjects involved in the extension action, focusing on the prominence and social participation of RAPS users, their relatives and healthcare personnel. In the interpretation of the extensionist action's results, the political meanings targeted in the relationship between the performance of the subjects involved and their everyday perceptions were emphasized. Therefore, the experience of the university extension could enable scholars and healthcare professionals, motivated by the tangible encounter with manifestations of social issues and by the theoretical thinking, the democratization of expertise and the formation with critical and ethical sense, with the prospect of playing a more specialized role to the discharge of mental health care services. To user's family members, proximity of the extension practice arouses as significant support to strengthen the protagonism in attention units, care and social participation in the public mental health policy.

KEYWORDS: Community extension, Community-institutional relations, Mental health services, Social participation.

\section{INTRODUÇÃO}

Os projetos de extensão universitária se constituem em importantes estratégias para ampliar os espaços de democratização do saber, de integração entre sociedade e universidade e de fortalecimento da relação entre ensino, pesquisa e extensão. Segundo a Política Nacional de Extensão Universitária (FORPROEX, 2015), a extensão universitária pode ser compreendida sob o princípio da indissociabilidade entre ensino, pesquisa e extensão, como "um processo interdisciplinar, educativo, cultural, científico e político que promove a

\begin{tabular}{l|l|l|l|l|l} 
Serv. Soc. \& Saúde & Campinas, SP & v. 20 & e021008 & 2021 & e-ISSN 2446-5992 \\
\hline
\end{tabular}


interação transformadora entre Universidade e outros setores da sociedade" (FORPROEX, 2015, p. 28).

A extensão universitária fundada nos princípios de uma educação transformadora, além de contribuir para a formação profissional e um conhecimento científico mais competente, potencializa a democratização do saber e gera um processo sistematizado de troca de saberes entre sociedade e universidade. Iniciativas educacionais embasadas em tal concepção podem contribuir para a reflexão e o aprimoramento das ações desenvolvidas em conjunto com a população e em espaços nos quais se desenvolve a proposta extensionista, como também para a própria academia e à docência, ao fomentar procedimentos pedagógicos renovados por uma perspectiva crítica, qualificada e compromissada, nas mais diversas dimensões da formação humana.

A aproximação com a abrangência comunitária propicia, no processo educativo, a elaboração da práxis e do saber científico e teórico, por meio da promoção de práticas vinculadas aos interesses e necessidades das populações mais vulnerabilizadas, social e economicamente.

No entanto, com a intenção de que o processo desenvolvido seja eficaz para a formação do/a estudante e igualmente para a comunidade, torna-se necessária a adoção de uma proposição de atuação pedagógica reflexiva, comprometida e engajada, ética e politicamente, a favor da promoção e garantia dos valores democráticos e da emancipação humana.

Essa compreensão permite refletir sobre a extensão universitária em seus princípios fundantes como força de resistência e potencial transformador, voltados ao protagonismo e ao fortalecimento da atenção psicossocial. No âmbito pedagógico e epistemológico, a reflexão relativa à extensão universitária se reporta à tradição pedagógica da educação, nos moldes da obra de Paulo Freire (1987), e à modalidade da investigação-ação-participação, segundo leituras de Borda (2015) e Thiollent (2002).

Essa concepção de extensão se caracteriza por um processo crítico e dialógico, integrado por proposta de formação universitária solidária e comprometida com transformações das sociedades, processo no qual educadores e educandos são transformados na dinâmica da práxis. Esse modelo é oposto ao formato de extensão universitária por transferência de conhecimentos, difusão cultural, divulgação científica e assistência tecnológica, em que os usuários seriam meramente receptores passivos em relação às

\begin{tabular}{|l|l|l|l|l|l} 
Serv. Soc. \& Saúde & Campinas, SP & v. 20 & e021008 & 2021 & e-ISSN 2446-5992 \\
\hline
\end{tabular}


informações e técnicas transmitidas (ARAUJO, 2018; FRAGA, 2017; TOMMASINO, CANO, 2016; MELO NETO, 2014).

Nessa direção caminham as reflexões compiladas no presente manuscrito, no sentido de trazer subsídios para se pensar metodologias participativas no contexto da política pública de saúde mental, a partir de um projeto extensionista, com envolvimento diversificado de distintos sujeitos: usuários de serviços de saúde mental, seus familiares, profissionais de saúde, docentes e estudantes.

Este artigo tem amparo nos resultados do projeto de extensão universitária intitulado "Processos formativos e participativos para o fortalecimento da Rede de Atenção Psicossocial e do protagonismo de usuários e profissionais", desenvolvido pelo Observatório de Saúde Mental da Universidade de Brasília(OBSAM/UnB). O projeto foi constituído por uma equipe multiprofissional e realizado no âmbito da Rede de Atenção Psicossocial (RAPS) do Distrito Federal (DF), Brasil, no período de agosto de 2018 a dezembro de 2019.

Nele foram desenvolvidos processos de capacitação voltados ao protagonismo de usuários, seus familiares e profissionais da saúde mental, nos espaços potenciais de participação social da RAPS do Distrito Federal, e dos movimentos sociais no campo da saúde mental no DF, orientados pelas seguintes proposições: a) consolidar os vínculos entre usuários, seus familiares e profissionais da saúde mental, no âmbito da RAPS-DF; b) dialogar sobre o contexto da política de saúde mental e da política pública de saúde, no período de realização do estudo, na instância do sistema oficial de saúde de país, o Sistema Único de Saúde (SUS), e no tocante aos seus rebatimentos nos processos de mobilização e participação social no âmbito da RAPS; c) instrumentalizar usuários, seus familiares e profissionais de saúde para a participação social qualificada em espaços representativos das políticas públicas e em movimentos sociais da sociedade civil; e d) contribuir para fortalecer o protagonismo dos usuários e de seus familiares nos espaços de atenção, cuidado e participação social, no âmbito da política pública de saúde mental em curso.

A atividade extensionista, com interface em pesquisa, vinculou-se a uma pesquisa de maior amplitude, intitulada "Reorganização dos e nos processos de trabalho na Rede de Atenção Psicossocial”, mediada pela avaliação participativa, aprovada pelo Comitê de Ética em Pesquisa (CEP) da Faculdade de Saúde da Universidade de Brasília, por meio do Protocolo ${ }^{\circ}$ 67425917.6.0000.0030, parecer $n^{\circ} 2.200 .022$, de 3 de agosto de 2017 . 
Desde as lutas e disputas travadas por pessoas, organizações da sociedade brasileira e trabalhadores da saúde mental, no contexto da promulgação da Constituição Federal do Brasil, de 1988, foram introduzidos dispositivos inovadores na rede de serviços da atenção à saúde mental, pautados por uma proposta antimanicomial, a exemplo dos Centros de Atenção Psicossocial (CAPS).

Os CAPS foram constituídos oficialmente pelo Ministério da Saúde, no ano de 1992, sendo regulamentados em 2002 como integrantes da rede de serviços de saúde no âmbito do SUS. O objetivo da criação dos CAPS foi prestar atendimento sistemático e constante aos indivíduos portadores de transtornos mentais severos e persistentes, em territórios com características específicas no tocante à saúde mental de suas populações, além de proporcionar cuidados clínicos e de reabilitação psicossocial. O desenvolvimento dessa modalidade de atenção à saúde mental se vinculou ao projeto de substituição do modelo tecnológico hospitalocêntrico, com o propósito de reduzir ou evitar internações e, ainda, com a perspectiva de favorecer o exercício da cidadania e da inclusão social dos usuários e respectivas famílias (BRASIL, 2004).

Entretanto, o fortalecimento de forças conservadoras e reacionárias, em contexto de acirramento do projeto neoliberal, tem operado uma contrarreforma psiquiátrica no Brasil, por meio de um processo de redução orçamentária, remanicomialização e mercantilização das ações e serviços de saúde mental (COSTA; MENDES, 2020).

Essa contrarreforma, na contramão das lutas do movimento antimanicomial, aprofunda-se por meio de ampliação do repasse orçamentário aos hospitais psiquiátricos. Na área de álcool e outras drogas, amplia-se o favorecimento das chamadas comunidades terapêuticas, nas quais vêm sendo identificadas influências de religiosidade de cunho fundamentalista e de medidas de rigor disciplinar.

Em outros termos: a contrarreforma retoma a força histórica do complexo médico psiquiátrico privatista mercantil na gestão das políticas e, além disso, ascende o crescimento e o favorecimento do conservadorismo e fundamentalismo religioso e suas interlocuções com o Estado (COSTA; MENDES, 2020), especialmente desde meados da segunda década do século XXI, no caso brasileiro.

Diante de tais considerações, empreendeu-se o presente estudo, com o objetivo de refletir sobre a contribuição de um projeto de extensão ao processo de formação educacional em saúde no país, empreendido no âmbito da Rede de Atenção Psicossocial do Distrito

\begin{tabular}{l|l|l|l|l|l}
\hline Serv. Soc. \& Saúde & Campinas, SP & v. 20 & e021008 & 2021 & e-ISSN 2446-5992 \\
\hline
\end{tabular}


Federal e com os aportes teórico-metodológicos de sustentabilidade do projeto. Pretendeuse enfatizar, neste artigo, as estratégias de mobilização e participação social observadas no projeto extensionista, no sentido de identificar os significados políticos, os conhecimentos e formas alternativas de organização social, constituídas a partir das vivências dos usuários e seus familiares, na vida cotidiana.

Buscou-se analisar a extensão em seus princípios, como força da resistência e potencialidade para contribuir para os processos formativos e participativos, no campo da saúde mental no SUS, no contexto da crise capitalista que tem acirrado o desmonte do Sistema de Proteção Social e do Sistema Único de Saúde, cujos efeitos, por conseguinte, incidem no enfraquecimento e na desconstrução da Reforma Psiquiátrica brasileira.

\section{Procedimentos metodológicos}

O estudo de que trata este artigo foi desenvolvido com abordagem qualitativa e ancorado na perspectiva da pesquisa-ação, de atuação participativa, constituída na interação e colaboração com os diferentes sujeitos no processo investigativo da realidade a ser trabalhada, na qual pesquisadores e envolvidos na investigação atuam de forma participativa e cooperativa, no enfrentamento de uma situação ou problema que lhes seja comum (THIOLLENT, 2011). O autor assim se expressa, em termos do desenvolvimento da pesquisa-ação em um projeto de extensão:

[...] a pesquisa-ação é realizada em um espaço de interlocução no qual os atores implicados participam na resolução dos problemas, com conhecimentos diferenciados, propondo soluções e aprendendo na ação. Nesse espaço, os pesquisadores, extensionistas e consultores exercem um papel articulador e facilitador em contato com os interessados (THIOLLENT, 2002, p. 67).

$\mathrm{Na}$ elaboração do presente artigo, foram utilizados recursos metodológicos das pesquisas bibliográfica e documental, como subsídio à discussão e à análise interpretativa dos resultados da pesquisa-ação do projeto de extensão; os diários de campo com os registros, observações e análises; material audiovisual produzido durante o acompanhamento e registro das atividades desenvolvidas nos CAPS; e, também, relatos documentais de outras ações empreendidas no decorrer do projeto.

Cabe destacar que a equipe extensionista foi constituída por estudantes de graduação, pós-graduação e docentes de várias áreas de formação, dentre as quais: Enfermagem, Serviço 
Social, Psicologia, Terapia Ocupacional e Saúde Coletiva, com o objetivo de criar pontes com as equipes dos CAPS e outros atores estratégicos. Dessa maneira, buscou-se, em diferentes momentos, a construção de um coletivo efetivo, ora integrado pela equipe extensionista, ora ampliado com os usuários e seus familiares, profissionais de saúde e militantes da saúde mental, nos processos formativos e participativos presentes nas ações desenvolvidas.

$\mathrm{Na}$ implementação do projeto de extensão e na pesquisa-ação, o alinhamento de cunho teórico-conceitual e de conteúdo metodológico ocorreu no movimento de práxis, envolvendo a equipe e demais sujeitos partícipes da proposta, por meio de estratégias de inserção em campo, especialmente nos CAPS, a exemplo das oficinas, seminários, debates e rodas de conversa, abarcando diferentes cenários de formação e intervenção.

$\mathrm{Na}$ sequência, estão apresentadas, em síntese, as atividades que estruturaram o desenvolvimento da atividade extensionista.

1. Centros de Atenção Psicossocial (CAPS) de todas as regiões de saúde do Distrito Federal:

Participação em reunião com a equipe de profissionais dos CAPS; oficina de cidadania; assembleias, grupos de usuários e familiares, grupos de ajuda mútua; oficina de geração de renda; organização e execução de cine-debate rodas de conversa; oficinas sobre participação social e controle social na política de saúde.

2. Centro Público de Economia Solidária:

Planejamento, execução e participação no Grupo de Trabalho de economia solidária e saúde mental e oficina "Economia Solidária e Saúde Mental”.

3. Universidade de Brasília (UnB):

Oficina de alinhamento conceitual/metodológico com vista à sistematização dos registros e documentação, com a equipe do projeto de extensão; planejamento e execução da oficina "Protagonismo de usuários e familiares: cidadania, participação social e saúde mental"; roda de conversa sobre saúde mental e democracia na atualidade, com participação de usuários, familiares e profissionais da RAPS; e oficina "Diálogos e construção de estratégias de enfrentamento à privatização da Política de Saúde Mental”. 


\section{Câmara Legislativa do Distrito Federal:}

Participação na Audiência Pública "A saúde mental no Distrito Federal”.

5. Fundação Oswaldo Cruz (Fiocruz) Brasília-DF:

Em parceria com o Centro Brasileiro de Estudos em Saúde (Cebes) e Movimento Pró-Saúde Mental, planejado e realizado o encontro com delegados da Etapa Distrital da $16^{\text {a }}$ Conferência Nacional de Saúde.

Em todas essas ações foram produzidas sínteses descritivas que no decorrer do projeto permitiram a avaliação da atividade em si, replanejamento das que viriam sucessivamente e que, depois de analisadas, viabilizaram a vi são da amplitude da proposta, no seu conjunto.

Nesse percurso, lançou-se mão de diários de campo, relatórios, material audiovisual produzido nas oficinas e em outras atividades grupais e houve, também, produção de músicas e poesias. Ressalta-se que a audiência pública teve participação significativa de usuários e familiares no que diz respeito não só à quantidade de participantes, mas à sua qualificação nas intervenções.

$\mathrm{Na}$ interpretação dos dados, foram consideradas categorias teóricas definidas pela equipe no contexto do projeto de extensão, além de categorias empíricas identificadas no processo de análise dos dados, com destaque para o estabelecimento dos conceitos de autonomia, participação, protagonismo e vínculo. As considerações teóricas e conceituais referidas ao conceito de extensão, delineadas na parte introdutória deste artigo, foram embasadas em análise da literatura.

$\mathrm{Na}$ análise interpretativa dos resultados da pesquisa-ação, com apoio de aportes teóricos incorporados no decorrer do projeto extensionista e complementados por subsídios conceituais da extensão universitária, coletados na literatura, foram realçadas as contribuições a respeito do desenvolvimento de processos formativos voltados ao fortalecimento do protagonismo de usuários e familiares da Rede de Atenção Psicossocial do Distrito Federal e, ainda, os significados políticos identificados na relação entre a atuação dos sujeitos envolvidos e suas vivências da vida cotidiana. 


\section{Extensão universitária e processos participativos em saúde mental}

Os recursos metodológicos adotados no projeto de extensão foram selecionados de modo a favorecer a interatividade entre usuários, familiares, equipe extensionista e profissionais de saúde no âmbito da Rede de Atenção Psicossocial (RAPS), com ênfase nos Centros de Atenção Psicossocial (CAPS). A metodologia participativa e dialogada, construída a partir das experiências dos sujeitos participantes, primou por dinâmicas de estímulo à autonomia, à participação e ao protagonismo de usuários e familiares.

As ações específicas de fomento da autonomia, protagonismo e participação dos usuários e familiares constituíram o eixo central da proposta de trabalho, com destaque para duas dimensões: a metodológica e a político-organizativa coletiva.

A dimensão metodológica refere-se ao planejamento, organização e realização das atividades desenvolvidas com usuários, familiares e profissionais, nas quais buscou-se desenvolver o uso de diferentes recursos de estímulo à participação daquelas pessoas na organização e realização das atividades. A dimensão político-organizativa coletiva refere-se ao fomento e estímulo do protagonismo e participação dos usuários e familiares em instâncias de defesa de direitos. Para tanto, essas dimensões abarcaram mobilizações e articulações com os movimentos de luta antimanicomial, com instâncias institucionalizadas de participação social e audiências públicas que ganharam destaque.

Identificou-se, no processo de articulação e mobilização dos usuários e familiares, a imprescindibilidade da constituição de vínculo entre a equipe extensionista, as equipes das unidades de serviços, usuários e familiares, no sentido da perspectiva teórico-metodológica adotada, com vistas a potencializar o protagonismo e a participação dos usuários e familiares.

Os CAPS se constituíram como espaços politicamente expressivos, com o propósito de representar ambientes de vinculação, aprendizado e compartilhamento de saberes. Numa via de mão dupla, o projeto permitiu experiências coletivas de formação e aprendizagem com usuários, familiares e profissionais dos CAPS e, também, com os estudantes e docentes envolvidos no projeto de extensão. A aproximação com as situações concretas vivenciadas pelos usuários e familiares da RAPS e suas repercussões no processo saúde-doença exigiram um processo investigativo-interventivo crítico, com nitidez da direção política engendrada na conjuntura contraditória e das forças econômicas e sociais em disputa.

\begin{tabular}{|l|l|l|l|l|l|} 
Serv. Soc. \& Saúde & Campinas, SP & v. 20 & e021008 & 2021 & e-ISSN 2446-5992
\end{tabular}


A metodologia participativa adotada foi fundada no compartilhamento de conhecimentos e aprendizagem, sob inspiração da obra de Paulo Freire (1987), segundo a qual "ninguém educa ninguém, ninguém educa a si mesmo, os homens se educam entre si, mediatizados pelo mundo" (FREIRE, 1987).

Nesse sentido, os contatos e a articulação com sujeitos e instituições identificadas como possíveis parceiras no projeto, as visitas institucionais, diálogos com profissionais, usuários e familiares, participações em reuniões do CAPS com a equipe ou durante as atividades terapêuticas grupais, ou, ainda, nas assembleias de usuários, dentre outras ações, assumem um componente crítico reflexivo, em contraposição a uma abordagem imediatista e burocratizada. Essas práticas, revestidas de caráter educativo, caracterizam-se como indutoras de processos participativos democráticos, observados em vários momentos do projeto.

O conceito de 'autonomia' foi interpretado como central no desenvolvimento das atividades, ao se considerar a sua transversalidade com os objetivos da Reforma Psiquiátrica, ou seja, "a construção da autonomia transcende o acesso a serviços sanitários e mesmo o acesso a políticas de saúde, desdobrando-se em produção de vida em articulação com redes de suporte social formais e informais, espaços de convivência, trabalho, lazer, cultura e arte, bem como o acesso a políticas públicas e direitos de cidadania" (AMARANTE; TORRE, 2018, p. 1096).

O 'protagonismo' foi compreendido como construção coletiva da saída da condição de "usuário-objeto" para a situação de "usuário-ator", sujeito político (AMARANTE, TORRE, 2018), alinhando-se ao conceito de 'cidadão', enquanto detentor da capacidade de participar da vida social e política (MATOS; SERAPIONE, 2017).

Igualmente central no escopo das atividades desenvolvidas no projeto de extensão e nas etapas da pesquisa-ação, a 'participação social' foi interpretada como espaço de permanentes contradições e conflitos, incapaz, por si só, de efetivar transformação social, mas com possibilidade de contribuir no adensamento e no fortalecimento da força política das classes populares, na configuração de repertórios, de mobilização e organização sociopolítica. A 'participação social' pode assegurar dispositivos e estratégias na construção de direitos, por meio de diversas iniciativas formais ou informais (fóruns, conselhos, associações e outros) (AMARANTE; TORRE, 2018).

Segundo essa visão, a participação social é compreendida como um processo de,

\begin{tabular}{|l|l|l|l|l|l} 
Serv. Soc. \& Saúde & Campinas, SP & v. 20 & e021008 & 2021 & e-ISSN 2446-5992 \\
\hline
\end{tabular}


[...] afirmação de singularidades possíveis, forjado nos encontros, parcerias, embates e discussões que se dão no cotidiano do trabalho em saúde, tanto nos espaços instituídos formais, criados para que as pessoas participem levando suas reivindicações e delegando poderes, quanto nos encontros que compõem o dia a dia da vida de um usuário da saúde mental em um Centro de Convivência Diária, como efeito dos processos instituintes que aí possam se produzir (COSTA; PAULON, 2012, p. 576577).

Nessa direção, a ampliação da participação do usuário em processos deliberativos, como definição do próprio Plano terapêutico sobre a organização das rotinas com a equipe do CAPS, participação em assembleias do Conselho de Saúde Local e/ou conferências de saúde mental, pode ter, do ponto de vista da micropolítica do processo de cuidado, a mesma potência transformadora de vidas (COSTA; PAULON, 2012, p. 576-577).

As atividades realizadas no âmbito do projeto de extensão suscitaram, por um lado, o protagonismo e a participação dos diferentes sujeitos envolvidos. Por outro lado, apesar da relevância de diferentes iniciativas, ainda prevalece um descompasso no movimento interacional usuário-família-profissional de saúde-universidade, não somente na temporalidade e disponibilidade dos envolvidos, mas especialmente nas condições objetivas de participação, ou, indo mais além, diante das investidas das políticas neoliberais e seus rebatimentos nos processos políticos e organizativos de coletivos.

A tradição democrática e popular, desde a década de 1990, sofre incursões das contrarreformas neoliberais, tendo adquirido novos contornos com a crise econômica mundial de 2008 por força de medidas de austeridade fiscal aplicadas nas políticas sociais engendradas em um contexto de crise política, econômica e social, sobretudo no Brasil, com o golpe jurídico-parlamentar em 2016, e no período pós-eleição de 2018, com a posse do presidente Jair Bolsonaro.

Os retrocessos implementados nas diferentes políticas sociais, sobretudo no SUS e na política pública de saúde mental, incidem diretamente na Rede de Atenção Psicossocial e nas estratégias de mobilização e participação social. Observa-se o projeto de Reforma Psiquiátrica, de bases antimanicomiais, agonizar em meio ao favorecimento das privatizações e ao fortalecimento de forças conservadoras e reacionárias (VASCONCELOS, 2020).

Considerando tal contexto, as ações do projeto de extensão, apesar de apresentarem condições potenciais de suscitar nos estudantes e profissionais de saúde uma formação pautada por senso crítico e ético, com perspectivas de contribuir de modo mais qualificado

\begin{tabular}{l|l|l|l|l|l}
\hline Serv. Soc. \& Saúde & Campinas, SP & v. 20 & e021008 & 2021 & e-ISSN 2446-5992 \\
\hline
\end{tabular}


para o fazer dos serviços de atenção em saúde mental, foram, por outro lado, fragilizadas pelas condições conjunturais desfavoráveis a tal propósito. Em relação aos usuários e familiares, embora o projeto de extensão tenha tido condições de potencializar o protagonismo nos espaços de atenção, cuidado e participação social na política de saúde mental, tal protagonismo também foi restringido por elementos adversos de natureza similar.

Nesse sentido, foi imprescindível introduzir a análise conjuntural no âmbito das ações realizadas no processo em questão. A abordagem elegida partiu de uma perspectiva de análise de conjuntura do cotidiano, compreendendo os acontecimentos, contextos, relações de força, a fim de se saber onde e como atuar (MARTINELLI, 2006). Sem se fechar no espaço cotidiano em si, a compreensão adotada foi de que as determinações conjunturais se expressam na vida cotidiana dos usuários e familiares de usuários dos serviços de saúde mental.

A concepção político-pedagógica selecionada para o desenvolvimento de atividades, a exemplo do planejamento participativo, oficinas, rodas de conversa, participação em audiências públicas, gerou oportunidades de espaços de reflexão sobre a conjuntura de retrocesso das políticas sociais e de análise crítica das medidas praticadas nas políticas públicas de saúde do país desde o golpe jurídico-parlamentar de 2016: o reforço ao autoritarismo e austerismo, o conservadorismo e a corrosão dos fundamentos democráticopopulares do SUS e, consequentemente, das conquistas da Reforma Psiquiátrica brasileira.

Diante da constatação de retrocessos das políticas de saúde mental, sobretudo no contexto da regressão de direitos sociais, e do aprofundamento das políticas neoliberais e neoconservadoras empreendidas no Brasil, especialmente nos governos de Michel Temer e de Jair Bolsonaro, as ações de mobilização coletiva e de protestos se ressignificaram. Novas acepções assumidas por mobilizações e protestos foram qualificadas como força de restabelecimento do debate na formulação de políticas de saúde mental, no âmbito do SUS, e de resistência ao crescimento de modelos ultraliberais e seus efeitos na desestruturação da Rede de Atenção Psicossocial, na privatização e mercantilização da saúde e no acirramento das desigualdades sociais. 


\section{CONSIDERAÇÕES FINAIS}

$\mathrm{Na}$ pesquisa-ação do projeto, empreendida por meio da atividade extensionista e apoiada por uma abordagem inter e transdisciplinar, foi priorizada a busca de conhecimentos para transcender intervenções, focalizadas e reducionistas, de atenção e cuidado à saúde mental. Os processos metodológicos adotados se pautaram na interatividade entre usuários, familiares, equipe extensionista e profissionais de saúde, compreendendo essa população como potenciais impulsionadores de processos participativos e promotores da autonomia e da emancipação humana.

Os resultados sugerem a necessidade de se renovar estratégias participativas na interrelação universidade-serviços de saúde mental e comunidade, tendo em vista a conjuntura em curso nas políticas sociais e, mais especificamente, na política pública de saúde mental. Esse caminho renovador exige um entrelaçamento entres os diferentes saberes, mas, sobretudo, disposição dos profissionais para a apropriação de pressupostos éticopolíticos críticos, fundantes de práticas comprometidas com a democracia, autonomia e emancipação humana.

O reforço à lógica neoliberal na política de saúde mental foi compreendido, nas várias dimensões do projeto de extensão e da pesquisa-ação, como um componente impeditivo no tocante ao envolvimento dos sujeitos em atividades coletivas participativas. Além de colocar em processo agonizante a política pública de saúde mental de bases antimanicomiais, o aprofundamento de medidas neoliberais e de austeridade fiscal fragilizam as políticas de proteção social e, por conseguinte, afetam diretamente as condições de vida e trabalho dos usuários e familiares, aumentam a segregação e dificultam o protagonismo e a autonomia dos diferentes sujeitos na participação em espaços coletivos de organização, na área da saúde mental. Para os usuários da política pública de saúde mental e seus familiares, além das singularidades presentes na condição de saúde desses indivíduos, a vulnerabilidade social e econômica influi diretamente no processo de cidadania e de participação.

As discussões a respeito da política de saúde mental, em contexto de acirramento das políticas neoliberais e da mercantilização da saúde, suscitaram reflexões no sentido da imprescindibilidade da organização sociopolítica dos usuários, familiares, movimentos sociais e profissionais da RAPS, com o propósito de instituir resistência às incursões dos setores privados lucrativos de mercantilização da saúde e das políticas públicas, em detrimento da perspectiva de direto social. As ações do projeto de extensão, ao constituírem 
um processo de investigação-ação em colaboração com os sujeitos de pesquisa, possibilitaram desenvolver experiências participativas e democráticas no fazer da investigação, com decisões coletivas e compartilhadas, entre os diferentes sujeitos do processo.

Recebido em abril de 2021 -Aprovado em novembro de 2021

\section{REFERÊNCIAS}

AMARANTE, P. D. de. C.; TORRE, E. H. G. “De volta à cidade, sr. cidadão!” Reforma Psiquiátrica e participação social: do isolamento institucional ao movimento antimanicomial. Rev. Adm. Pública. Rio de Janeiro, n. 52, n. 6, p. 1090-1107, nov./dez. 2018. Disponível em: https://www.scielo.br/scielo.php?script=sci_arttext\&pid=S003476122018000601090\&lang $=$ pt Acesso em: 17 fev. 2021.

ARAÚJO, R. S. de. Extensão popular: horizontes teórico-metodológicos para a condução do trabalho social universitário orientado pela educação popular. In: ARAÚJO, R. S. de; CRUZ, P. J. S. C. (Org). Educação popular e práticas sociais: Ação, processo formativo e construção do conhecimento Editora do CCTA, 2018. p. 49-99. Disponível em: http://www.ccm.ufpb.br/vepopsus/wpcontent/uploads/2019/01/EDUCA\%C3\%87\%C3\%83O-POPULAR-E-PR\%C3\%81TICASSOCIAIS-Editora-do-CCTA-2018.pdf. Acesso em: 12 fev. 2021.

BRASIL. Ministério da Saúde. Secretaria de Atenção à Saúde. Departamento de Ações Programáticas Estratégicas. Saúde mental no SUS: os centros de atenção psicossocial. Brasília: Ministério da Saúde, 2004. 86 p. (Série F. Comunicação e Educação em Saúde). Disponível em: http://www.ccs.saude.gov.br/saude_mental/pdf/sm_sus.pdf. Acesso em: 4 fev. 2021.

COSTA, D. F. C. da; PAULON, S. M. Participação Social e protagonismo em saúde mental: a insurgência de um coletivo. Saúde debate, v. 36, n. 95, p. 572-582, Rio de Janeiro, dez. 2012. Disponível em: https://www.scielo.br/scielo.php?script=sci_arttext\&pid=S010311042012000400009\&lng=pt\&nrm=iso\&tlng=pt. Acesso em: 17 fev. 2021.

\begin{tabular}{|l|l|l|l|l|r}
\hline Serv. Soc. \& Saúde & Campinas, SP & v. 20 & e021008 & 2021 & e-ISSN 2446-5992 \\
\hline
\end{tabular}


COSTA, P. H. A. da.; MENDES, K. T. Contribuição à crítica da economia política da contrarreforma psiquiátrica brasileira. Argumentum, v. 12, n. 2, p. 44-59, Vitória, mai./ago. 2020. Disponível em:

https://periodicos.ufes.br/argumentum/article/view/28943/21383. Acesso em: 17 fev. 2021. FALS BORDA, O. Cómo investigar la realidad para transformarla. In: FALS BORDA, O. Una sociología sentipensante para América Latina. México, D.F.: Siglo XXI Editores; Buenos Aires: CLACSO, 2015. p. 253-6. Disponible en: http://biblioteca.clacso.edu.ar/clacso/se/20151027053622/AntologiaFalsBorda.pdf. Acceso en: 23 feb. 2021.

FORPROEX. Fórum de Pró-Reitores das Instituições Públicas de Educação Superior Brasileiras. Política Nacional de Extensão Universitária, Manaus-AM, maio de 2012. 68 p. Florianópolis: Universidade Federal de Santa Catarina, UFSC, 2015. Disponível em: https://proex.ufsc.br/files/2016/04/Pol\%C3\%ADtica-Nacional-de-Extens\%C3\%A3oUniversit\%C3\%A1ria-e-book.pdf. Acesso em: 15 jan. 2021.

FRAGA, L. S. Transferência de conhecimento e suas armadilhas na extensão universitária brasileira. Avaliação (Campinas), v. 22, n. 2, p. 403-419, Sorocaba, ago. 2017. Disponível em:

https://www.scielo.br/scielo.php?script=sci_arttext\&pid=S141440772017000200403\&lng $=$ pt\&nrm=iso\&tlng=pt. Acesso em: 14 fev. 2021.

FREIRE, P. Pedagogia do oprimido. 17. ed. Rio de Janeiro: Paz e Terra, 1987.

MARTINELLI, M. L Reflexões sobre o Serviço Social e o projeto ético-político profissional. Emancipação, v. 6, n. 1, p. 9-26, Ponta Grossa, 2006. Disponível em: https://dialnet.unirioja.es/revista/15555/V/6. Acesso em: 17 fev. 2021.

MELO NETO, J. F. de. Extensão popular. 2. ed. João Pessoa: Editora da UFPB, 2014. 122 p. Disponível em: https://www.ufpb.br/redepopsaude/contents/biblioteca-1/extensaopopular/extensao-popular.pdf. Acesso em: 23 fev. 2021.

MATOS, A. R.; SERAPIONI, M. O desafio da participação cidadã nos sistemas de saúde do Sul da Europa: uma revisão da literatura. Cad. Saúde Pública, v. 33, n. 1, e00066716, Rio de Janeiro, jan. 2017. Disponível em: https://www.scielo.br/scielo.php?script=sci_arttext\&pid=S0102311X2017000102002\&lng=pt\&nrm=iso\&tlng=pt. Acesso em: 16 fev. 2021. THIOLLENT, M. Metodologia da pesquisa-ação. 18. ed. São Paulo: Cortez, 2011. 
THIOLLENT, M. Construção do conhecimento e metodologia da extensão. Revista Cronos, v. 3, n. 2, p. 65-71, Natal-RN, jul/dez; 2002. [Publicado on line: 10 out. 2018]. Disponível em: https://periodicos.ufrn.br/cronos/article/view/15654/10730. Acesso em: 12 fev. 2021.

TOMMASINO, H; CANO, A. Modelos de extensión universitaria en las universidades latinoamericanas en el siglo XXI: tendencias y controversias. Universidades, n. 67, p. 724, Ciudad de México, enero/marzo 2016. Disponible en: https://www.redalyc.org/pdf/373/37344015003.pdf. Acesso en: 13 feb. 2021.

VASCONCELOS, E. M. Reforma Psiquiátrica, tempos sombrios e resistência: diálogos com o marxismo e o serviço social. Campinas: Papel Social, 2016.

VASCONCELOS, E. M. A gravidade do neoliberalismo radical pós 2008 e nossas estratégias de resistência. Argumentum, v. 12, n. 2, p. 47-66, Vitória, mai./ago. 2020. Disponível em: https://periodicos.ufes.br/argumentum/article/view/30483/21380. Acesso em: 17 fev. 2021. 